\title{
Pleomorphic Adenoma of Parotid Gland - Clinical Case in 14-Years-Old Child
}

\author{
Mahammad M Davudov* and Parvin Hasanova \\ Department of Oral and Maxillofacial Surgery, Azerbaijan Medical University, Baku, Azerbaijan
}

\begin{abstract}
Pleomorphic adenomas of salivary glands in children's ages is one of the rare finds. These tumors are 1-3\% of the head and neck tumors. The most common pleomorphic adenoma of the salivary gland is found in the parotid (60-65\%). This slowly growing tumor is most commonly seen in women in the $5^{\text {th }}-6^{\text {th }}$ decades. Histologically, these tumors include epithelial, mesenchymal, myoepithelial components covered by pseudocapsulus. Pleomorphic adenoma is found to be very late as $1-1.5 \%$ for children aged 16 and under [1].
\end{abstract}

\section{Introduction}

Salivary glands tumors are found in $1-3 \%$ of the head and neck tumors. About $60-70 \%$ of these tumors are seen in parotid, $70-80 \%$ of them are adenomas [2]. These tumors are more commonly seen in the age group of 40 to 50 years, with $1-5 \%$ in the age of 16 years or older.

Pleomorphic adenoma's cell contents include epidermoid and myoepithelial cells. If these cells are different from each other, the corresponding adducts are tumors. If these two cells are identical, the adjunctive adjunct to adjuvant is pleomorphic adenoma. Pleomo-adenoma etiology is a wellknown, benign tumor and epithelial and mesenchymal differentiation. The epithelial part develops from myoepithelial cells, which, when developed from the channel epithelium, provide mezentral partial contraction. That is, the pleomorphic term is used not for pleomorphism of the cells, but for architectural pleomorphism [3].

These tumors, most commonly occurring in the parotid, develop from the surface and are felt in a non-symptomatic mass. About $10 \%$ of pleomorphic adenomas develop from deeper levels, and compression to the medial is assessed as mass in parafaringeal edema, with intraabdominal swelling.

Chang and Lee reported that the age range of salivary gland tumors was 1-76 and the mean age of benign birth defects was 36.6 . Only $2-5 \%$ of them occur in patients under the age of 16. Pleomorphic adenoma is found to be less than $10 \%$ in children. For this reason, the clinical case we publish is rarely seen as localization and age [4].

\section{Case Report}

A 14-year-old boy was admitted to our clinic with a complaint of swollen lymph nodes. From the history of the patient, it has been known for almost a year now that the swelling is present in the early years of its existence, has grown over time but has never been anxious about it. The patient's clinical examination revealed a mild, rigid, elastic, painless skin with accurate boundaries in the left parotid area. There were no problems with the face symptoms of the facial nerve, internal organs - the opening of the mouth (Figure 1).

The patient was sent to the MRI examination and the result of the examination was a homogeneous, derivative of the right parotid gland located on the rear compartment (Figure 2).

We found a patient with a modification Blair incision under general anesthesia. And superficial parotidectomy was performed taking into account the risk of recurrence. Macroscopically no pathological tissue was observed in the nerve branches. The patient was given intradermal suture with a $5 / 0$ prolene for minimal cosmetic deformation. As a result of the histopathological examination of the lesion, pleomorphic adenoma diagnosis was confirmed. Normal postoperative wound healing was observed. No signs of facial paresis were observed (Figure 3).

Histopathology: Pleomorphic adenoma of parotid gland. The derivative has a thin capsule. Stroma has a slim structure in large fibrous sites and a small area of chondroid component. $\mathrm{HE} \times 100$.

*Corresponding author: Mahammad M Davudov, Department of Oral and Maxillofacial Surgery, Azerbaijan Medical University, Baku, Azerbaijan

Accepted: September 11, 2019

Published online: September 13, 2019

Citation: Davudov MM, Hasanova P (2019) Pleomorphic Adenoma of Parotid Gland - Clinical Case in 14-Years-Old Child. J Head Neck Surg 2(1):29-32 


\section{Discussion}

Tumors of the salivary glands of glandular origin are rare in the pediatric age group, and only about $1.7 \%$ of all

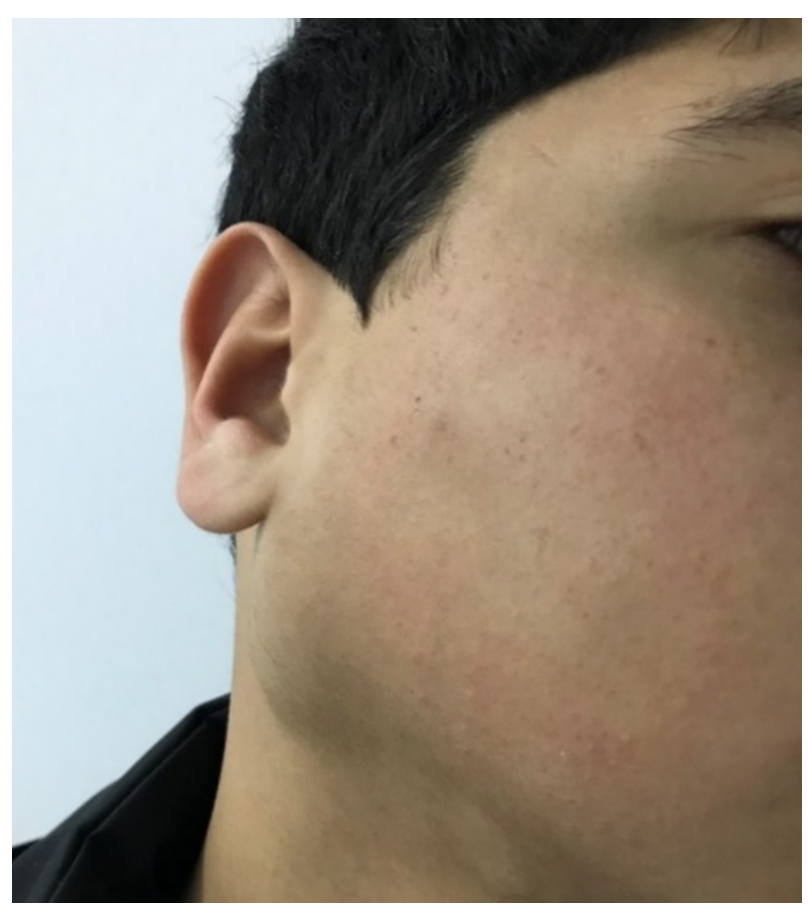

Figure 1: No problems with the face symptoms of the facial nerve, internal organs - the opening of the mouth. epithelial saliva tumors are found in children [5]. In addition, masses arising in pediatric salivary glands are more likely to consist of vascular anomalies, such as hemangiomas and lymph node malformations or infectious and inflammatory lymphadenopathy, rather than glandular tumors of salivary origin. When real epithelial tumors of the glands arise, their extremely low frequency often prevents any single pediatric institution from reporting the exact treatment algorithm. Thus, the same protocols used to treat adult salivary gland tumors are often used for children. It is believed that the behavior of the main salivary gland tumors in children is associated with a histological type and clinical degree, similarly to adults [6]. Approximately $50 \%$ of all parotid masses in the pediatric age group are malignant neoplasms, which is higher than in adults $[7,8]$. As in adults, the pleomorphic adenoma is the most common tumor of the glandular epithelial gland $[9,10]$. The most common malignant neoplasm of the glands in children is mucoepidermoid carcinoma, followed by adenocarcinoma and acino-cell carcinoma [7,8]. When children have mucoepidermoid carcinoma of the parotid gland, the histological evaluation is usually low and the longterm outcome with appropriate treatment is favorable. When all pediatric saliva masses are examined (and not just those for whom a parotidectomy sample was obtained), recent reports suggest that benign lesions predominate in the vast majority. For example, in a series of cases from the Massachusetts infirmary for eyes and ears of 22 children who had an unknown continuous parotid mass for 8 years, only one patient had a malignant neoplasm (mucoepidermoid carcinoma) [5]. Eight

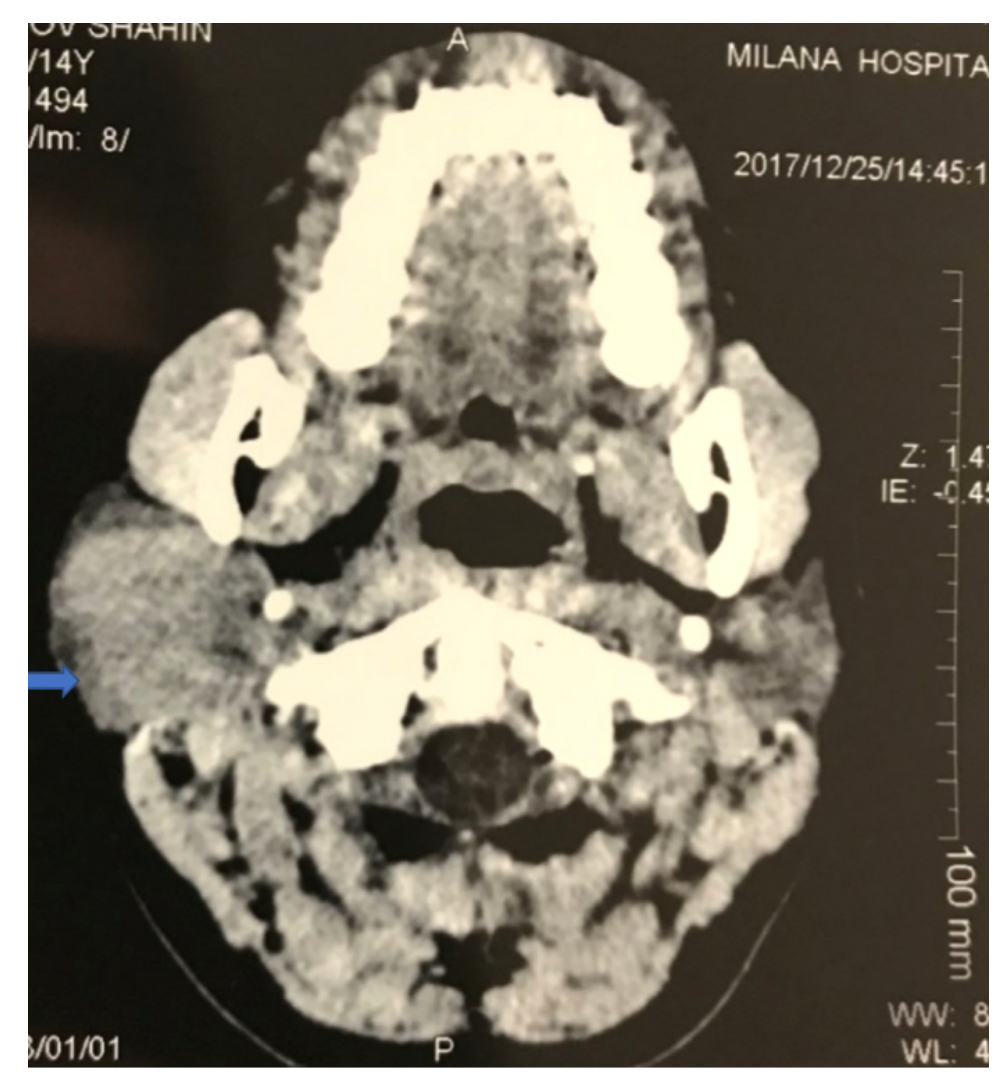

Figure 2: MRI examination and the result of the examination was a homogeneous, derivative of the right parotid gland located on the rear compartment. 


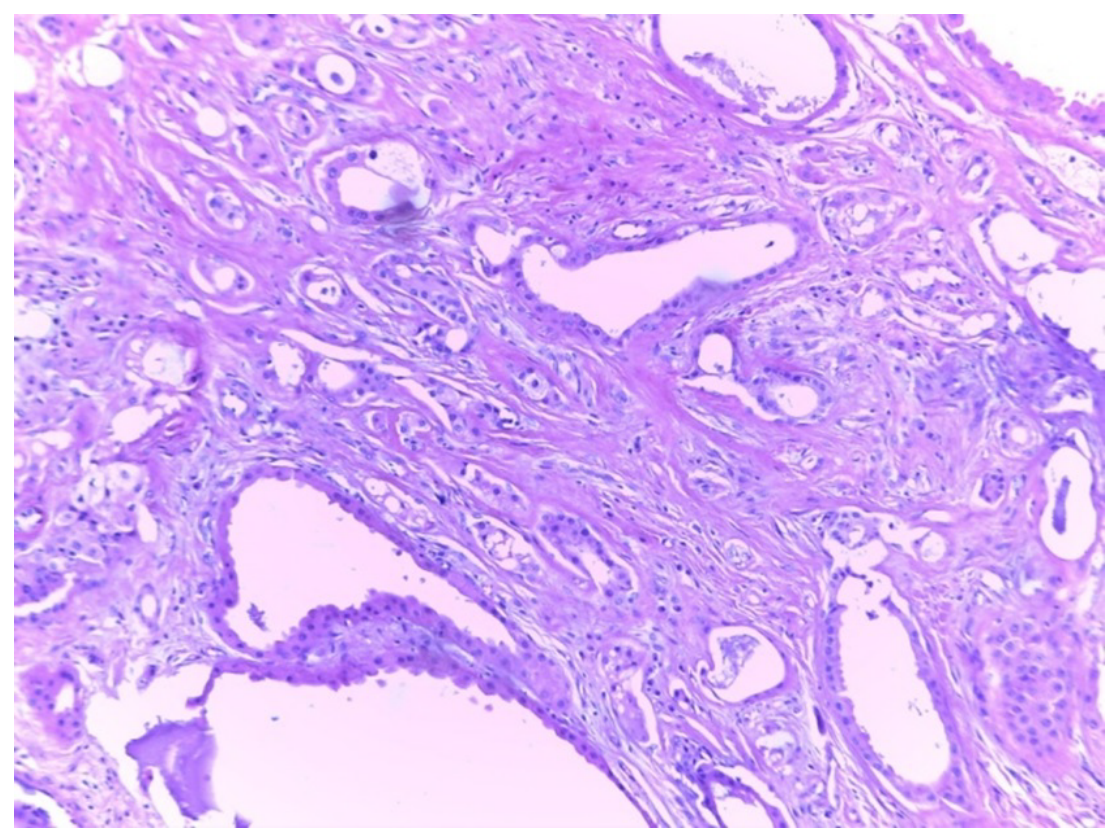

Figure 3: Histopathology: Pleomorphic adenoma of parotid gland.

patients (36\%) had pleomorphic adenoma, and 13 patients (59\%) had an inflammatory process, such as feline scratching, non-tuberculous mycobacterial infection or toxoplasmosis. In a survey of all 118 children who underwent surgical treatment of the parotid mass from 1970 to 1997 at the Mayo Clinic in Rochester, Minnesota, $84 \%$ had benign lesions, while only (16\%) had malignant tumors [6]. The most common benign parotid mass was a pleomorphic adenoma $(48 \%$ of benign tumors). Other benign lesions included hemangiomas, gill slit cysts, lymphatic malformations and neurofibromas. If vascular lesions were excluded, parotid gland tumors were malignant in $35 \%$ of patients. Since more recent parotid hemangiomas were observed (and not dissected) in later years of research, the number of parotid hemangiomas included in the analysis was probably underestimated. In a recent study of 324 masses in the region of the salivary glands from the Children's Memorial Hospital in Chicago, 87\% of all masses of the salivary glands in children were vascular anomalies (59\% of all masses were hemangiomas and $27.5 \%$ were lymph node defects) [1]. Only 43 patients (13\%) had solid masses, and most benign solid salivary masses did not have a glandular origin, but rather non-salivary tumors and inflammatory processes were included. Only 10 of 324 of all pediatric salivary formations (3\%) were malignant tumors, the most common was low-grade mucoepidermoid carcinoma with subsequent rhabdomyosarcoma.

\section{Acknowledgments} study.

We are grateful to patient who participated in the

\section{Funding}

The study was supported by Azerbaijan Medical University.

\section{Authorship}

$\mathrm{MMD}$ and $\mathrm{PH}$ were the main investigators. $\mathrm{PH}$ collected the data and wrote the first draft. All authors read and approved the manuscript.

\section{Conflict of Interest Statement}

The ethics committees of Azerbaijan Medical University approved the study.

\section{Consent for Publication}

Not applicable.

\section{Competing Interests}

The authors declare that they have no competing interests.

\section{References}

1. Bentz BG, Hughes CA, Ludemann JP, et al. (2000) Masses of the salivary gland region in children. Arch Otolaryngol Head Neck Surg 126: 1435-1439.

2. Jagadishkumar K, Anilkumar MG, Krishna Kumar HC, et al. (2014) Pleomorphic adenoma of the cheek in a child: A case report. Dent Res J (Isfahan) 11: 522-524.

3. Eveson JW, Cawson RA (1985) Salivary gland tumours. A review of 2410 cases with particular reference to histological types, site, age and sex distribution. J Pathol 146: 51-58.

4. Chang EZ, Lee WC (1989) Surgical treatment of salivary gland tumors. J Oral Maxillofac Surg 47: 555-558.

5. Camacho AE, Goodman ML, Eavey RD (1989) Pathologic correlation of the unknown solid parotid mass in children. Otolaryngol Head Neck Surg 101: 566-571.

6. Orvidas L, Kasperbauer JL, Lewis JE, et al. (2000) Pediatric parotid masses. Arch Otolaryngol Head Neck Surg 126: 177-184. 

2(1):29-32

7. Krolls SO, Trodahl JN, Boyers RC (1972) Salivary gland lesions in children. A survey of 430 cases. Cancer 30: 459-469.

8. Ogata H, Ebihara S, Mukai K (1994) Salivary gland neoplasms in children. Jpn J Clin Oncol 24: 88-93.
9. Castro EB, Huvos AG, Strong EW (1972) Tumors of the major salivary glands in children. Cancer 29: 312-317.

10. Klem C, Mair EA (1999) Four-duct ligation: A simple and effective treatment for chronic aspiration from sialorrhea. Arch Otolaryngol Head Neck Surg 125: 796-800.

DOI: $10.36959 / 605 / 533$ 